\title{
Bendamustine/Obinutuzumab Regimen
}

National Cancer Institute

\section{Source}

National Cancer Institute. Bendamustine/Obinutuzumab Regimen. NCI Thesaurus. Code C154212.

A chemoimmunotherapy consisting of bendamustine and obinutuzumab that can be used in the treatment of some forms of non-Hodgkin lymphoma (NHL). 